\title{
Erratum to: Using Polarization to Control the Phase of Spatial Modes for Application in Quantum Information
}

\author{
W. F. Balthazar • D. P. Caetano • C. E. R. Souza •
}

J. A. O. Huguenin

Published online: 13 November 2014

(C) Sociedade Brasileira de Física 2014

Erratum to: Braz J Phys (2014) 44:658-664

DOI 10.1007/s13538-014-0250-6

The correct affiliation for C. E. R. Souza is Instituto de Física, Universidade Federal Fluminense, Niterói, Rio de Janeiro, Brazil.

The online version of the original article can be found at http://dx.doi.org/ 10.1007/s13538-014-0250-6.

\section{W. F. Balthazar}

Programa de Pós Graduação em Física and Instituto de Ciências Exatas, Universidade Federal Fluminense, Volta Redonda, Rio de Janeiro, Brazil

e-mail: wagnerbalthazar@if.uff.br

D. P. Caetano

Escola de Engenharia Industrial Metalúrgica de Volta Redonda, Universidade Federal Fluminense, Volta Redonda, Rio de Janeiro, Brazil

e-mail: dpcaetano@id.uff.br

\section{J. A. O. Huguenin ( $\triangle)$}

Instituto de Ciências Exatas and Programa de Pós-Graduação em Física, Universidade Federal Fluminense, Niterói, Rio de Janeiro, Brazil

e-mail: jose_huguenin@id.uff.br

\section{E. R. Souza}

Instituto de Física, Universidade Federal Fluminense, Niterói, Rio de Janeiro, Brazil

e-mail: carloseduardosouza@id.uff.br 